\title{
APPORTS ET LIMITES DU MARQUAGE INDIVIDUEL DANS LA CONNAISSANCE DE L'ÉCOBIOLOGIE DU CHEVAINE (LEUCISCUS CEPHALUS).
}

\author{
H. LE LOUARN et J.L. BAGLINIÈRE
}

INRA, Laboratoire d'Écologie Aquatique, 65 rue de St-Brieuc, 35042 Rennes Cedex, France.

\section{RÉSUMÉ}

Depuis 1991, des marquages individuels ont été effectués sur des chevaines (Leuciscuss cephalus) de la rivière Boutonne, affluent de la Charente, afin d'améliorer les connaissances écobiologiques de l'espèce qui restent très fragmentaires pour les populations atlantiques françaises. 718 individus, de taille supérieure à $120 \mathrm{~mm}$, ont été capturés par pêche électrique sur un secteur de $2,7 \mathrm{~km}$, lors de la période de reproduction (mi-juin), et marqués essentiellement par marque nouille (5 années) mais également par implant visible (une année) durant cinq ans. Au total, 162 poissons ont été recapturés et certains individus plusieurs fois au cours de la période d'étude. L'analyse des résultats fait ressortir que :

- Seuls deux poissons marqués par implant visible ont été recapturés $(0,9 \%)$; le taux moyen de recapture des individus marqués par marque nouille a été de $22,3 \%$ mais a varié selon l'année et l'état de maturité du poisson (de 6,6 à $37,7 \%$ ) ; il apparaît plus faible pour les immatures $(6,4 \%)$ que pour les géniteurs $(25,2 \%)$ et reste identique chez les deux sexes.

- La méthode de capture-recapture a permis de valider l'estimation de l'âge et de la croissance par scalimétrie : définition de la marque hivernale; existence d'une chronologie de l'apparition de l'anneau hivernal et donc de la reprise de croissance; validation de la régression reliant la longueur du poisson à celle de son écaille pour la population étudiée.

- Les déplacements à l'intérieur du secteur étudié sont faibles et les femelles montrent une grande fidélité à leur site de reproduction.

- Le marquage par marque nouille entraîne un déficit de croissance, principalement chez les poissons de plus de cinq ans ; ce déficit semble se réduire dès la deuxième année suivant le marquage.

La discussion des résultats montre qu'en dépit de certains facteurs limitants, l'utilisation de cette technique de marquage individuel apparaît contribuer d'une manière significative à la connaissance de l'écobiologie du chevaine, allant jusqu'à montrer la possible existence de deux sous-populations sur le secteur étudié.

Mots-clés : chevaine, Leuciscus cephalus, marquage, validation âge et croissance, déplacement. 


\section{CONTRIBUTION AND LIMITS OF INDIVIDUAL MARKING TECHNIQUES FOR IMPROVEMENT IN THE ECOBIOLOGY KNOWLEDGE OF CHUB (LEUCISCUS CEPHALUS).}

\section{ABSTRACT}

Individual tagging experiments were carried out on chub, Leuciscus cephalus, from 1991 in the river Boutonne, a tributary of the river Charente. The aim of the study was to improve the ecobiological knowledge of this species in its Atlantic French distribution range. 718 chubs of more than $120 \mathrm{~mm}$ length were electrofished in a sector of $2.7 \mathrm{~km}$ length during spawning season (mid June) and tagged with floy-tags (5 years) and visible implants ( 1 year) during five years. 162 fishes were recaptured and some of them several times during the studied period. Main results showed that :

- Only 2 fishes tagged with visible implants were recaptured $(0.9 \%)$; the average recapture rate for the floy-tagged fishes was $22.3 \%$, changing according to year and sexual maturation state $(6.6$ to $37.7 \%)$; it was much lower in immature ones $(6.4 \%)$ than in spawners $(25.2 \%)$ and was similar for males and females.

- Capture-recapture method allowed to validate age and growth estimation by scalereading : good winter band definition; timing of winter band appearance and beginning of the new growing season; reliable relationship between fish length and scale size for the studied population.

- Movements inside the studied area were low, especially in females (no movement observed).

- Tagging by floy-tags resulted in a depressed growth rate, especially in older fishes (more than 5 years). This impact disappeared the second year after tagging.

Results were discussed and showed that, despite some restricting factors, individual tagging allowed to improve many ecobiological knowledges in chub, up to a possible discrimination of the population into two sub-populations spatially separated in the studied sector.

Key-words : chub, Leuciscus cephalus, tagging, age and growth validation, movement.

\section{INTRODUCTION}

Le chevaine (Leuciscus cephalus Linné, 1766) possède une aire de répartition très large sur le continent euro-asiatique, couvrant une partie de la Russie et du Proche-Orient (BLANC et al., 1971). Il est donc largement répandu dans toute l'Europe, à l'exception du nord de la Scandinavie, du sud de l'Italie, de l'Écosse et de l'Irlande (RICHARD, 1973). A la périphérie de son aire de distribution, principalement dans les régions méridionales, l'espèce se subdivise en un certain nombre de sous-espèces (SPILLMANN, 1961; BIANCO et RECCHIA, 1983). En France, ce poisson est présent partout, sauf dans l'ouest de la Bretagne (ALLARDI et KEITH, 1991), essentiellement sous la forme $L$. cephalus cephalus, la deuxième sous-espèce (L. cephalus cabeda) n'existant que dans le Midi (SPILLMANN, 1961).

Le chevaine, au même titre que la vandoise (Leuciscus leuciscus), est considéré comme un cyprin d'eaux vives ou rhéophile (ARRIGNON, 1972 ; PHILIPPART, 1972). Son habitat est étendu et correspond essentiellement aux zones à ombres et à barbeaux de HUET (1949), même s'il peut être trouvé dans la zone à truites (TIMMERMANS, 1957). De fait, cette espèce, de faible valeur économique et surtout recherchée pour la pêche de loisir, semble à même de coloniser un grand nombre de milieux et occuper, actuellement, une place importante dans les écosystèmes aquatiques. 
La quasi-totalité des travaux réalisés sur la biologie voire la dynamique des populations de cette espèce, au demeurant peu nombreux, a été effectuée en dehors des frontières françaises (lles britanniques: HELLAWELL (1971), HICKLEY et BAILEY (1982); Belgique : PHILIPPART (1972); Italie : VITALI et BRAGHIERI (1984); Tchécoslovaquie : PROKES (1981), HANEL (1982); Croatie : HABEKOVIC et al. (1993). Seul RICHARD (1973) l'a étudiée sur la Seine.

Depuis 1977, le Laboratoire d'Écologie Aquatique (INRA de Rennes) a entrepris des études sur les caractéristiques des peuplements de poissons sur quelques cours d'eau de la façade atlantique française classés en deuxième catégorie piscicole (Canut, Flume) ou en limite de première catégorie (Colmont, Boutonne). Ces rivières possèdent des communautés pisciaires différentes mais largement dominées par le chevaine, ce qui a permis de récolter un certain nombre de données biologiques sur cette espèce. Cette récolte n'a pas été homogène dans la mesure où les données n'ont pas été recueillies aux mêmes périodes et où tous les aspects écologiques n'ont pas été abordés sur tous les cours d'eau (LE LOUARN et al., 1997). Cependant, depuis 1990, un suivi annuel régulier du peuplement pisciaire et notamment des populations de truites, de brochets, de vandoises et de chevaines a été entrepris sur la Boutonne. Outre l'analyse des relations entre ces populations en termes de compétition et de prédation, et celle des potentialités truiticoles du cours d'eau, l'objectif de cette étude était l'amélioration des connaissances sur la biologie et l'écologie du chevaine. Pour ce faire, des marquages individuels et un suivi de la population de géniteurs ont été effectués lors de la période de reproduction du chevaine (mi-juin) pendant cinq ans. Hormis l'étude réalisée par LIBOSVARSKY (1966) avec un marquage de groupe, l'espèce n'avait fait l'objet d'aucune investigation de ce type jusqu'à présent. Les résultats de ce travail sont présentés en essayant de dégager les aspects biologiques nouveaux apportés par cette technique d'étude, tout en analysant ses avantages et ses limites d'utilisation en milieu naturel.

\section{SITE ET PÉRIODE D'ÉTUDE}

La Boutonne est un affluent rive droite de la Charente, long de $98 \mathrm{~km}$ pour une superficie de bassin versant de $1030 \mathrm{~km}^{2}$. Sa pente moyenne est de $0,14 \%$ et son débit moyen annuel de $5,95 \mathrm{~m}^{3} / \mathrm{sec}$. Ses caractéristiques hydroécologiques, essentiellement karstiques et liées à un réseau aquifère, lui confèrent un régime hydrologique complexe marqué par les apports de sources mais aussi par des débits d'étiage faibles (milieu de l'été).

Trois éléments permettent de caractériser la physicochimie de l'eau de cette rivière :

- une amplitude thermique annuelle moyenne $\left(7,5^{\circ} \mathrm{C}\right.$ à $\left.19^{\circ} \mathrm{C}\right)$; cependant, certaines années, la température de l'eau peut atteindre 23 à $24^{\circ} \mathrm{C}$ les mois les plus chauds ;

- une très grande transparence d'eau;

- une teneur élevée en ion $\mathrm{Ca}++(106$ à $119 \mathrm{mg} / \mathrm{l})$.

Les activités humaines sur le bassin versant sont essentiellement tournées vers l'agriculture (élevage et culture du maïs) dont la consommation en eau n'a fait qu'augmenter ces dernières années (arrosages importants dès le mois d'avril). La qualité de l'eau reste bonne à acceptable.

La Boutonne est un cours d'eau non-domanial classé en première catégorie de sa source à St-Jean-d'Angely et domanial de deuxième catégorie en aval.

La station étudiée est située dans le cours moyen de la rivière ( $1^{\text {re }}$ catégorie) entre deux moulins (Châtre et Le Port, département de la Charente-Maritime) (Fig. 1) ; sa longueur est de $2700 \mathrm{~m}$ pour une surface en eau de $27500 \mathrm{~m}^{2}$. Elle est constituée d'une 
majorité d'habitats de type plat, entrecoupés de profonds (3 à $4 \mathrm{~m}$ ) et/ou de radiers (zones courantes moins profondes), les plus importants d'entre eux étant situés aux abords des deux moulins ; les fonds sont en général très sableux.

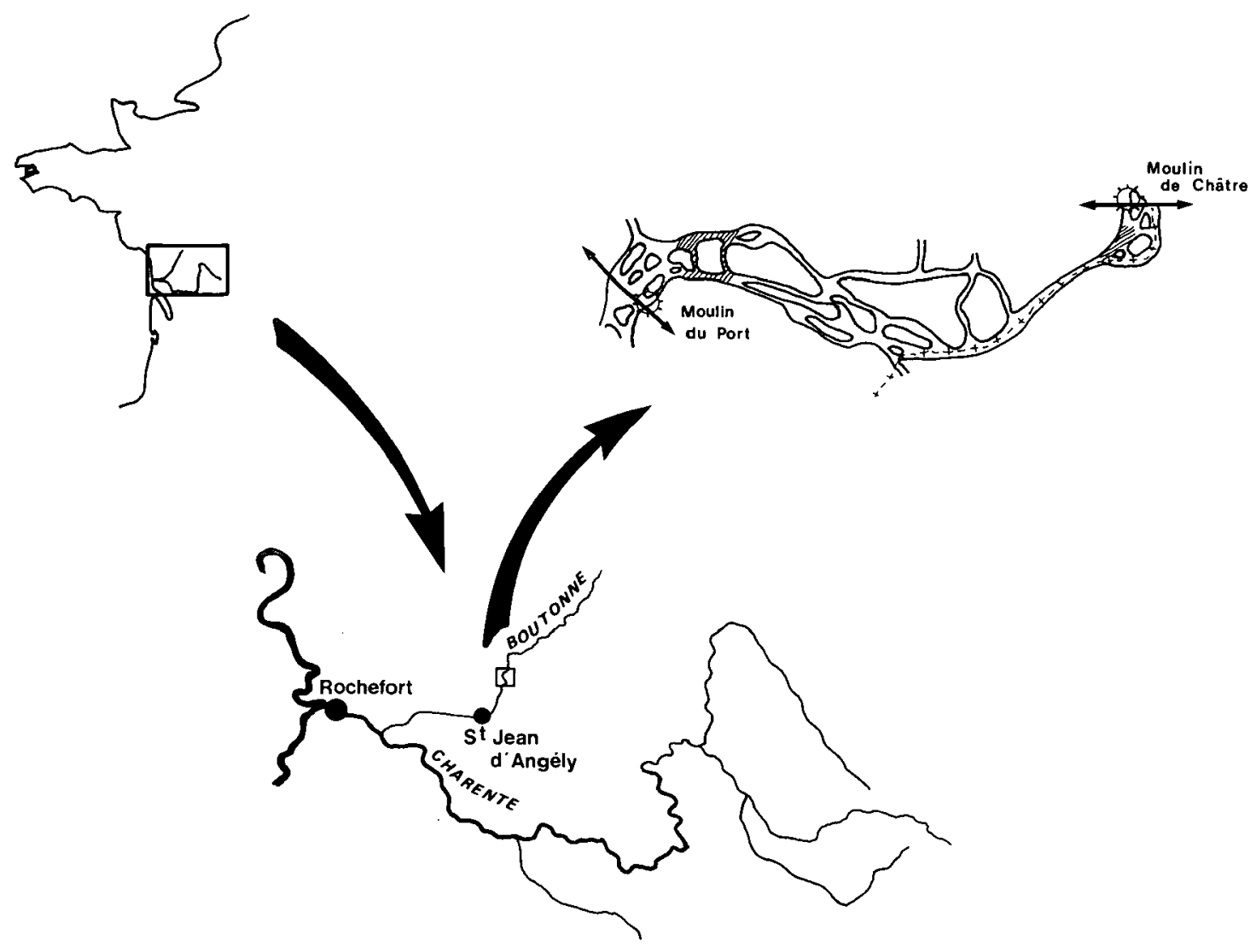

Figure 1

Secteur inventorié par pêche électrique sur la Boutonne de 1991 à 1996.

$\longleftrightarrow$ limites du secteur étudié

$\square Z Z Z 7$ lieux de reproduction

\section{Figure 1}

Electrofishing area in the river Boutonne from 1991 to 1996. studied sector boundaries spawning areas

Les campagnes de pêche ont toujours eu lieu lors de la saison de reproduction du chevaine, soit entre le début et la troisième semaine du mois de juin (Tableau I). Cependant, l'activité de reproduction était plus ou moins importante selon les conditions météorologiques ayant prévalu au début du printemps et, certaines années, ces campagnes ont pu se situer soit juste avant soit à la fin de la phase active de reproduction. Ainsi, selon les conditions hydrologiques, des concentrations de poissons ont pu être observées sur certains secteurs particuliers. En revanche, des regroupements importants de chevaines ont toujours été notés sur les zones de radiers proches des moulins limitant à l'aval et à l'amont le secteur d'étude. 


\section{Tableau 1}

Périodes des campagnes de pêche réalisées sur la Boutonne de 1991 à 1996. Relation avec la saison de reproduction du chevaine et type de marquage effectué (FT : marque nouille ; IV : implant visible).

\section{Table I}

Electrofishing census periods made in the Boutonne from 1991 to 1996. Relationship with chub spawning-season and used tagging type (FT : floy-tag ; IV : visible implant).

\begin{tabular}{|c|c|c|c|}
\hline Année & Date & Reproduction & Marquage \\
\hline 1991 & $03-05 / 06$ & début & FT \\
1992 & $15-17 / 06$ & en cours en aval & FT \\
1993 & $14-15 / 06$ & en début & FT \\
1994 & $13-15 / 06$ & en cours & FT \\
1995 & $12-14 / 06$ & en début & IV \\
1996 & $24-26 / 06$ & terminée & IV et FT \\
\hline
\end{tabular}

\section{TECHNIQUES ET MÉTHODES}

\section{Récolte des données}

Tous les poissons ont été échantillonnés par pêche électrique réalisée soit en bateau, soit à pied. Les populations ont été essentiellement recensées à l'aide de sondages (un seul passage) en raison des grandes largeurs et/ou profondeurs de la rivière; seuls deux secteurs (radier et bras courant) ont fait l'objet, chaque année, d'un inventaire (deux passages).

Tous les poissons ont été mesurés ( $L f$, longueur à la fourche en $\mathrm{mm}$ ). Pour une très grande majorité d'entre eux, le poids a été noté ( $\mathrm{P}$ en $\mathrm{g}$ ) et des écailles ont été prélevées sur une zone située entre la nageoire dorsale et la ligne latérale. Le sexe et l'état de maturité ont été identifiés à partir de critères externes (boutons de noce et spermiation chez les mâles, ovulation chez les femelles).

A partir de 1991, des marquages ont été effectués sur les poissons d'une taille supérieure à $120 \mathrm{~mm}$ à l'aide d'une marque nouille numérotée et placée en-dessous de la nageoire dorsale (DELL, 1968). En 1995, des implants visibles numérotés (film de polyester de dimensions $2,5 \mathrm{~mm} \times 0,9 \mathrm{~mm} \times 0,13 \mathrm{~mm}$ ) ont été introduits à l'aide d'un trocart sous l'épiderme transparent de la bordure postérieure du globe oculaire (HAW et al., 1990). La validité de ce type de marque est actuellement testée expérimentalement en étang sur un certain nombre d'espèces (chevaine, brochet, perche).

Les recaptures ont été faites essentiellement par pêche électrique les années suivant la campagne de marquage mais également par la pêche sportive (dans ce cas, possibilité de recapture l'année du marquage). 


\section{Traitement des données}

Une relation linéaire entre la longueur du poisson et celle de son écaille a été établie.

L'analyse des poissons recapturés marqués a permis d'identifier la marque d'arrêt de croissance (marque hivernale) et de comparer la croissance observée à celle calculée à partir des relations de rétromesures. Afin d'homogénéiser les lectures scalimétriques (poissons pris à différentes périodes), l'âge de tous les poissons a été donné en comptabilisant le nombre de marques hivernales sans qu'il soit suivi du signe conventionnel + , même si le poisson avait tout juste repris sa croissance.

La reprise de croissance: a été estimée par comptage des circuli formés après la dernière marque hivernale présente sur l'écaille et par comparaison avec le nombre moyen de stries formées au cours d'une année complète. Cette estimation a été faite à partir d'échantillons de poissons capturés soit à la même période durant plusieurs années, soit durant plusieurs mois au cours d'une même année (cas des reprises faites par la pêche à la ligne).

Le taux de recapture est calculé pour l'année de marquage en tenant compte de tous les individus recapturés, y compris ceux repris plusieurs fois au cours de la période d'étude. De plus, il a été exprimé en tenant compte du sexe du poisson et de son état de maturité. Ainsi, trois classes ont été définies : mâle, femelle et immature. Cette dernière catégorie comprend des immatures vrais mais également des individus n'ayant pas été déterminés sexuellement à l'époque du recensement.

Le taux de croissance moyen annuel d'une classe d'âge (TC) a été calculé en faisant la moyenne des croissances des individus composant cette classe d'âge et en séparant les poissons recapturés marqués des non-marqués. Ce taux de croissance individuel (TCl) a été exprimé par la formule suivante: $T C l=L_{i+1}-L_{i} / L_{i} \times 100$ avec $L_{i}$ et $L_{i+1}$ représentant la taille d'un individu aux années $\mathrm{i}$ et $\mathrm{i}+1$.

La comparaison des données a été faite à l'aide de tests statistiques classiques (t de Student, écart réduit, $\mathrm{Chi}^{2}$ ).

\section{RÉSULTATS}

\section{Nombre et caractéristiques des poissons recapturés (Tableau II)}

718 chevaines ont été marqués par marque nouille, donnant lieu à 162 reprises dont 41 multiples et dans 6 cas à plusieurs années d'intervalle, soit un taux de recapture moyen de $22,3 \%$ sur l'ensemble de la période d'étude. La taille des poissons recapturés s'étale de 181 à $460 \mathrm{~mm}$ pour un spectre d'âge de 3 à 13 ans.

Le taux de recapture varie fortement selon l'année de marquage $\left(p<10^{-3}\right)$, passant de $6,8 \%$ en 1992 à 37,7\% l'année d'après. Cette variation n'apparaît pas reliée au nombre de poissons marqués puisque la valeur la plus faible du taux de reprise est observée l'année où le nombre d'individus marqués est le plus élevé. De même, le taux de recapture varie fortement $\left(p<10^{-3}\right)$ selon l'état de maturité du poisson au marquage puisqu'il est en moyenne de $6,4 \%$ pour les immatures ou indéterminés et de $25,2 \%$ pour les géniteurs. Chez ces derniers, le taux de recapture est identique quel que soit le sexe.

La majorité des recaptures $(66,7 \%)$ s'effectue l'année suivant celle de marquage et dans ces conditions, le taux de recapture reste identique pour les deux sexes (Chi ${ }^{2}$ non significatif). Les reprises peuvent s'échelonner sur quatre années et le pourcentage de reprise à plusieurs années d'intervalle est identique pour les deux sexes, même s'il apparaît plus étalé dans le temps et plus élevé pour les femelles $(10,5 \%)$ que pour les mâles $(7,5 \%)$.

Sur 222 individus marqués par implants visibles, seules deux recaptures ont été enregistrées, soit un taux de reprise de $0,9 \%$. 


\section{Tableau II}

Évolution du taux de recapture annuel des chevaines marqués au cours de la période d'étude en fonction du sexe et de l'état de maturité ( $A$ : année de marquage ; $A+1$ : année suivant celle du marquage ; $A+2$ : deux années après le marquage ; ...).

\section{Table II}

Evolution of annual recapture rate in tagged chubs during the studied period according to sex and sexual maturation state ( $A$ : tagging year; $A+1$ : one year after tagging ; $A+2$ : two years after tagging ; ...).

\begin{tabular}{|c|c|c|c|c|c|c|c|c|c|}
\hline & \multirow{3}{*}{$\begin{array}{l}\text { Nombre de } \\
\text { poissons } \\
\text { marqués }\end{array}$} & \multicolumn{8}{|c|}{ Poissons recapturés } \\
\hline & & \multicolumn{2}{|c|}{$A+1$} & \multicolumn{2}{|c|}{$A+2$} & \multicolumn{2}{|c|}{$A+3$} & \multicolumn{2}{|c|}{$A+4$} \\
\hline & & $\mathbf{N}$ & $\%$ & $\mathbf{N}$ & $\%$ & $\mathbf{N}$ & $\%$ & $\mathbf{N}$ & $\%$ \\
\hline 1991 & & & & & & & & & \\
\hline Mâles & 20 & 1 & 5,0 & 1 & 5,0 & & & & \\
\hline Femelles & 13 & 1 & 7,6 & 1 & 7,5 & 1 & 7,6 & & \\
\hline 1992 & & & & & & & & & \\
\hline Mâles & 147 & 11 & 13,4 & 5 & 3,4 & 1 & 0,7 & & \\
\hline Femelles & 89 & 9 & 10,1 & 6 & 6,7 & 2 & 2,2 & 1 & 1,1 \\
\hline Immatures & 45 & & & & & 1 & 2,2 & & \\
\hline 1993 & & & & & & & & & \\
\hline Mâles & 81 & 21 & 25,9 & 10 & 12,3 & 4 & 4,9 & & \\
\hline Femelles & 49 & 12 & 24,5 & 4 & 8,2 & 2 & 4,1 & & \\
\hline Immatures & 13 & & & 1 & 7,6 & & & & \\
\hline 1994 & & & & & & & & & \\
\hline Mâles & 141 & 36 & 25,5 & 7 & 5,0 & & & & \\
\hline Femelles & 68 & 13 & 19,1 & 6 & 8,8 & & & & \\
\hline Immatures & 52 & 4 & 7,6 & 1 & 1,9 & & & & \\
\hline Total & & & & & & & & & \\
\hline Mâles & 389 & 65 & 17,7 & 23 & 5,6 & 5 & 1,3 & & \\
\hline Femelles & 219 & 35 & 16,0 & 17 & 7,8 & 5 & 2,2 & 1 & 0,5 \\
\hline Immatures & 110 & 4 & 3,4 & 2 & 1,7 & 1 & 0,8 & & \\
\hline
\end{tabular}

\section{Localisation des recaptures}

La très grande majorité des reprises (155/162, soit $95,7 \%$ ) s'est faite dans la zone de première capture. Sur les 7 déplacements observés, 6 sont effectués par des mâles et le dernier par un individu immature ou sexuellement non identifié ; 5 de ces déplacements se sont produits depuis la zone moyenne du secteur d'étude, constituée principalement de profonds voire de fosses, vers un des radiers délimitant le secteur et constituant une des zones de reproduction. Les mêmes observations ressortent des captures multiples, puisque seulement deux changements pour 41 poissons (un mâle et un immature) sont constatés. Enfin, les reprises à plusieurs années d'intervalle ont toutes eu lieu dans la zone de première capture. 


\section{Validation de l'âge et des rétromesures}

Sur les écailles de chevaine, les cycles annuels sont marqués par une alternance de zones claires et sombres correspondant à des périodes de croissance différentes. L'anneau hivernal ou la ligne d'arrêt de croissance apparaît, quelle que soit la population étudiée, comme une marque claire très nette, sauf pour la première année où elle reste toujours plus difficile à discerner. L'examen des écailles des poissons recapturés marqués individuellement permet de confirmer la structure de cette marque et de localiser son apparition dans le temps, entre fin mai et début juin. II montre également qu'il existe une excellente correspondance (1) du nombre d'anneaux hivernaux et de circuli formés les années précédentes, (2) de la taille mesurée et de celle rétrocalculée, (3) des tailles rétrocalculées à partir de l'écaille prélevée à la capture et à la recapture. Dans ce dernier cas, sur 33 séries de mesures, le pourcentage de déviation est toujours inférieur à 2,7\% sauf pour la taille au premier hiver $(4 \%)$, en raison du manque de netteté de la marque, notamment dans la diagonale de mesure.

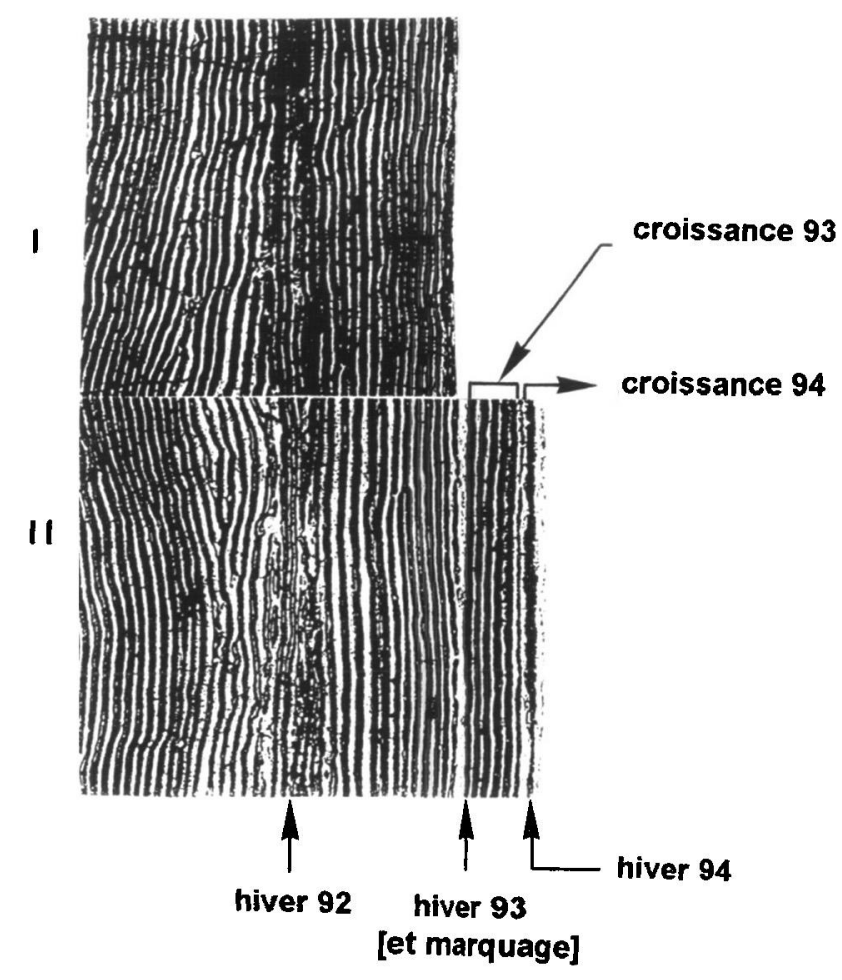

Figure 2

Validation des tailles rétromesurées chez un chevaine marqué en juin 1993 $(L f=293)$ et recapturé en juin $1994(L f=307)$ : tailles rétromesurées lors du marquage : 74, 172, 235, 255 ; tailles rétromesurées lors du contrôle : 74, 172, 234, 257,292 . Lors du marquage, la reprise de croissance 93 n'avait pas encore eu lieu. Lors de la recapture, la croissance 94 avait repris et l'hiver 94 était donc marqué. On note en outre une correspondance du nombre de circuli sur les deux écailles.

Figure 2

Validation of the back-calculated sizes in a chub tagged in June 1993 ( Lf = 293) and recaptured in June 1994 ( $L f=307$ ) : back-calculated sizes at tagging : 74,172 , 235, 255 ; back-calculated sizes at recapture : 74, 172, 234, 257, 292. At tagging time, growth recovery had not taken place yet. At recapture time, plus growth was observed and winter band was visible. Furthermore, we can observe a good correspondence between circuli number on the two scales. 
Cette excellente relation entre les modalités de croissance révélées aux âges successifs est parfaitement illustrée par la figure 2. Dans ce cas, sont accolées deux sections d'écailles du même poisson, l'une lors du marquage (I), l'autre lors de la reprise (II). Au marquage, en juin 1993, la croissance annúélle n'avait pas repris. Les observations du contrôle effectué en juin de l'année suivânte font apparaître :

- une configuration identique des circuli au cours des années 1992 et 1993 ;

- une croissance identique de l'écaille en 1993, avec dans l'un et l'autre cas formation de 17 circuli.

Cette très bonne correspondance générale entre les structures superficielles de l'écaille ainsi que les valeurs très élevées des coefficients de régressions linéaires entre la longueur du poisson et celle de son écaille (Tableau III) permettent de valider les modèles de rétromesure établis et, ainsi, de considérer que les valeurs de taille rétrocalculées sont semblables à celles observées sur les poissons de même âge capturés pour la première fois à la même époque.

\section{Tableau III}

Relation entre la longueur du poisson (Lf) et celle de son écaille (Le) pour la population de chevaine de la Boutonne (totale et géniteurs).

\section{Table III}

Relationship between fish length (Lf) and scale length (Le) for chub population (total and spawners) in the river Boutonne.

\begin{tabular}{|l|l|l|c|}
\hline Mâles & Lf $=0,407$ Le $+48,028$ & $r=0,89$ & $n=309$ \\
Femelles & Lf $=0,375$ Le $+95,96$ & $r=0,92$ & $n=139$ \\
Ensemble & Lf $=0,409$ Le $+42,149$ & $r=0,95$ & $n=691$ \\
\hline
\end{tabular}

\section{Limites d'utilisation du marquage}

Ces limites d'utilisation ne concernent que le marquage par marque nouille en raison du très faible taux de recapture de poissons marqués par implants visibles.

Le taux de perte de marque reste faible puisque seulement 15 individus, soit $2,1 \%$ de la population totale marquée, ont perdu leur marque. Cependant, la quasi-totalité de ces individus présentait des plaies vives ou en voie de cicatrisation et des écailles de régénération.

En revanche, ce type de marquage a un impact très sensible sur la croissance puisque celle-ci diminue fortement chez les poissons marqués. Ce phénomène est parfaitement illustré par la figure 2 (écaille inférieure) où seulement 6 circuli de croissance ont été formés pendant la saison 1994, entre la période de capture et.celle de recapture, pour un accroissement de taille de $7 \mathrm{~mm}$ (soit $+2,4 \%$ ). Cette observation est confortée par la comparaison des taux de croissance des poissons marqués (128 cas utilisables) avec ceux des,cohortes correspondantes avant marquage (Tableau.IV). Le déficit est toujours bien marqué, surtout à partir de la 5 ème année. Pour les poissons plus âgés, la croissance annuelle restant naturellement faible ne permet plus de comparaison fiable. 


\section{Tableau IV}

Comparaison entre les taux de croissance des chevaines marqués et non-marqués.

\section{Table IV}

Growth rate comparison between tagged and untagged chubs.

\begin{tabular}{|c|ccc|ccc|c|}
\hline Age & \multicolumn{3}{|c|}{ Poissons non-marqués } & \multicolumn{3}{|c|}{ Poissons marqués et } & Niveau de \\
recapturés & & \multicolumn{3}{c|}{$\begin{array}{c}\text { des } \\
\text { dification } \\
\text { différences }\end{array}$} \\
\hline $2-3$ & 584 & 51,7 & 22,5 & 12 & 13,4 & 11,2 & $\mathrm{p}<10^{-4}$ \\
\hline $3-4$ & 442 & 25,5 & 18,1 & 22 & 9,9 & 6,0 & $\mathrm{p}<10^{-4}$ \\
\hline $4-5$ & 256 & 14,3 & 7,3 & 24 & 4,9 & 4,2 & $\mathrm{p}<10^{-4}$ \\
\hline $5-6$ & 172 & 8,7 & 4,7 & 23 & 4,1 & 3,0 & $\mathrm{p}<10^{-4}$ \\
\hline $6-7$ & 96 & 6,1 & 2,7 & 20 & 2,6 & 1,0 & $\mathrm{p}<10^{-4}$ \\
\hline $7-8$ & 54 & 4,8 & 2,2 & 17 & 1,8 & 1,3 & $\mathrm{p}<10^{-4}$ \\
\hline $8-9$ & 25 & 4,3 & 1,9 & 6 & 2,3 & 1,9 & $\mathrm{p}<10^{-2}$ \\
\hline $9-10$ & 10 & 3,4 & 12,0 & 1 & 0,3 & & \\
\hline $10-11$ & 4 & 4,6 & 1,0 & 3 & 0,2 & 0,3 & $\mathrm{p}<10^{-4}$ \\
\hline
\end{tabular}

\section{DISCUSSION}

Le marquage de poissons, individuel ou de masse, est la plupart du temps associé à des programmes de recherche sur la biologie ou l'économie des pêches (PARKER et al., 1990). Très souvent, il sert à la caractérisation et au suivi de populations de poissons migrateurs. Ainsi, cette technique d'étude a été ou est encore largement employée chez les salmonidés (BAGLINIÈRE et al., 1987 ; MAISSE et al., 1987 ; BAGLINIĖRE et PORCHER, 1994), permettant des avancées intéressantes dans la connaissance de la biologie et de l'écologie de ces espèces en milieu aquatique continental (BAGLINIÈRE et al., 1989, 1993). En revanche, cette technique d'étude et de suivi des populations a été ou reste encore peu utilisée chez les autres poissons d'eau douce et notamment chez le chevaine qui reste une espèce de faible intérêt halieutique. En ce sens, l'étude réalisée sur les populations de cette espèce à l'aide de techniques de marquage individuel pallie cette lacune. Par ailleurs, elle permet d'approfondir l'écologie du chevaine tout en soulignant les limites voire les inconvénients inhérents à la technique de marquage utilisée.

\section{Apports sur les connaissances biologiques}

\section{Biologie générale}

Le nombre important de reprises et leur répartition au cours des années après marquage confirment la longévité du chevaine et notamment celle des femelles puisqu'une d'entre elles a été recapturée 4 ans après le marquage (LIBOSVARSKY, 1966 ; HELLAWELL, 1971 ; MANN, 1976 ; LE LOUARN et BAGLINIERE, 1996). 
En période de reproduction, on observe sur la Boutonne une ségrégation spatiale entre les géniteurs et les individus immatures : $24 \%$ des premiers sont localisés dans les zones profondes contre $94 \%$ des seconds. A cette époquee, les géniteurs sont concentrés sur les deux sites de reproduction situés en amont et en aval du secteur d'étude. Ces sites sont constitués par des bras courants et des zones de radiers de la rivière principale. L'habitat de ces milieux est caractérisé par des vitesses de courant élevées, un substrat mixte (du sable au roc) avec des abris à proximité (habitats profonds, sous-berges, végétation aquatique et/ ou surplombante).

En second lieu, au vu des résultats des opérations de marquage-recaptures, le chevaine (et plus particulièrement la femelle) semble montrer une très grande fidélité à son site de reproduction. En outre, cette grande fidélité du chevaine ayant atteint l'âge de maturité va au-delà du seul attachement à un site de reproduction puisqu'elle semble s'étendre à une zone plus large (mais restant relativement restreinte au niveau spatial), englobant également des habitats de stabulation et/ou d'alimentation. Dans ces conditions, au regard des résultats des marquages réalisés sur plus de six années (95,7\% de reprises dans la zone originelle de capture), il semble que l'on puisse émettre l'hypothèse de l'existence de deux sous-populations ayant des domaines vitaux séparés par une zone centrale de transition, avec néanmoins de possibles échanges mais qui resteraient très réduits. Cependant, cette hypothèse est tempérée par le faible taux de recapture des immatures qui semble indiquer une dispersion nettement plus importante pouvant aller jusqu'à une possible émigration en dehors du secteur étudié. Néanmoins, ces observations rejoignent celles faites par NICOLAS et al. (1994) qui montraient le partage de la population de chevaines présente sur le Bas-Rhône en deux stocks distincts, chacun d'entre eux étant constitué par deux composantes, dont l'une majoritaire était sédentaire.

En 1993, la campagne de pêche s'est déroulée alors que la saison de reproduction n'avait pas débuté. $37 \%$ des chevaines adultes ont été pêchés dans le secteur moyen constitué de profonds, dans le cours principal de la rivière, soit deux fois plus que les autres années. De plus, tous les immatures marqués au cours de l'étude dans ce même secteur ont été repris dans l'une ou l'autre des zones de radiers. On peut donc noter après SPILLMANN (1961), KIRKA (1965), HABASHY (1974), ECONOMOU et al. (1991) un déplacement lié à la reproduction, donc une véritable migration.

Ces déplacements sont certainement dépendants du niveau d'eau en hiver et au printemps. En 1992, année où le débit resta très faible, la population cantonnée en aval était particulièrement importante puisque 197 chevaines matures y furent marqués. L'année suivante, seulement 20 recaptures ont été effectuées, soit un taux de 10,1\% pour les femelles et $13,4 \%$ pour les mâles au lieu de 20 à $25 \%$ les autres années. On peut alors supposer, cette année-là, un apport extérieur de géniteurs provenant d'une population cantonnée plus en aval et dont une partie importante aurait participé temporairement au rassemblement de ponte du radier aval.

\section{Validation de l'estimation de l'âge et de la croissance}

En dépit de la grande facilité d'interprétation des écailles de chevaine (BAGLINIĖRE et LE LOUARN, 1987), l'estimation de l'âge et de la croissance par scalimétrie se doit d'être validée. L'utilisation de poissons marqués reste une des meilleures méthodes pour une telle validation et cette démarche a été généralement beaucoup plus employée chez les salmonidés que chez les autres espèces (OMBREDANE et BAGLINIĖRE, 1992). Ainsi, les résultats obtenus dans la présente étude sur le chevaine restent relativement novateurs puisqu'ils montrent que :

- La définition d'une manière claire de la marque hivernale et l'excellente adéquation entre ces arrêts hivernaux et le nombre de circuli observés au cours du temps sur les écailles des poissons recapturés marqués permettent de valider la méthode scalimétrique 
pour l'estimation de l'âge. Cependant, il reste un certain nombre de difficultés pour localiser le premier annulus, qui pourrait ne pas apparaître sur l'écaille en fin de saison de croissance (LE LOUARN et al., 1997).

- Il existe une chronologie de l'apparition de l'anneau hivernal et donc de la reprise de croissance qui se situe à la fin du printemps (mi-mai à début juillet) mais reste fonction de l'âge, du sexe et de l'état de maturité du poisson (LE LOUARN et BAGLINIËRE, 1996). De telles observations ont déjà été faites, mais d'une manière beaucoup moins précise, d'une part sur la période de la reprise de croissance (CRAGG-HINE et JONES, 1969; HELLAWELL, 1971 ; PHILIPPART, 1972) et d'autre part sur sa variabilité d'apparition dans le temps avec l'âge (sous-espèce $L$. cephalus cabeda, VITALI et BRAGHIERI, 1984).

- La validation des modalités de la croissance est possible à partir de l'examen des écailles des poissons recapturés marqués en raison des excellentes régressions reliant la longueur du poisson à celle de son écaille et malgré la très forte baisse du taux de croissance induite par le marquage. Néanmoins, pour approfondir cette validation de l'estimation de la croissance à partir des écailles à l'ensemble de la population, il serait nécessaire (1) d'augmenter la gamme de taille pour les relations longueur du poisson longueur de l'écaille, (2) de marquer des poissons de petite taille, ce qui n'est pas possible avec les deux types de marques utilisés à ce jour.

\section{Limites d'utilisation des marquages}

L'utilisation de la technique de marquage par marque nouille présente deux inconvénients :

- Le premier est lié au fait que cette marque ne peut être apposée sur des poissons de taille inférieure à $120 \mathrm{~mm}$, sous peine d'une forte mortalité. Ainsi, il y a toute une partie de la population et notamment les juvéniles qui ne peut être suivie en utilisant cette technique. Cette relation type de marquage - taille du poisson est confirmée par un taux de recapture enregistré pour les poissons immatures ou indéterminés sexuellement quatre fois plus faible que celui des géniteurs. Par ailleurs, il apparaît que cette catégorie de poissons est surtout constituée d'individus immatures (73\%) au vu de l'âge et de la taille de première maturité du chevaine estimés pour la population de la Boutonne (LE LOUARN et al., 1997). Ainsi, cette différence entre les taux de reprise peut s'expliquer par la petite tálle des individus immatures, qui les rend difficiles à capturer ou à recapturer mais également par leur plus grande dispersion (y compris peut-être en dehors du secteur d'étude) comparée au regroupement des géniteurs dans les zones de radiers à l'époque du recensement.

- Le deuxième inconvénient provient de l'impact du marquage par marque nouille sur la croissance et la survie individuelle. La présence de plaies non cicatrisées observées sur certains individus un an après le marquage laisse penser que cette technique peut influer sur le taux de survie du poisson et cela d'autant plus que ce dernier est manipulé à une période où il est certainement le plus fragile. Néanmoins, le très faible nombre de poissons présentant ce type de plaie ou de cicatrice $(1,9 \%)$ et le taux moyen de recapture élevé des géniteurs observé sur toute la période d'étude tempèrent cette supposition d'impact élevé du marquage sur la survie.

En revanche, le marquage par marque nouille a une réelle influence sur la croissance du poisson durant la première année suivant le marquage. Ainsi, il est impossible de prendre en compte l'estimation de la croissance postérieure au marquage, même si les conséquences semblent disparaître au bout d'un an. Les écailles des poissons recapturés ne peuvent plus être prises comme structures de référence pour l'analyse de la croissance de l'ensemble de la population (BEAMISH et MCFARLANE, 1983). A titre de comparaison, il faut noter que ce type de marquage (marque nouille) ne semble pas affecter la croissance des salmonidés dans la gamme de taille observée dans 
l'étude. Ainsi, les effets sont tout juste significatifs chez la truite arc-en-ciel (MCALLISTER et al., 1992) et sont nuls chez la truite de mer (BERG et BERG, 1987). Enfin, des marquages par marque nouille ont été réalisés simultanément sur les populations de truites et de brochets de la Boutonne, sans qu'ait été enregistré un quelconque impact sur leur croissance. Par ailleurs, cette abseñce d'impact chez le brochet, espèce possédant des écailles de grande taille (un pèu moins grandes cependant que celles du chevaine), ne permet pas d'envisager l'hypothèse du développement d'un foyer d'infection à partir de la suppression d'une surface importante d'épiderme. II semble plus logique d'évoquer un phénomène de stress lié au marquage chez le chevaine, espèce à croissance lente, et d'autant plus accentué que l'individu est âgé.

Les limites d'utilisation de la technique par marque nouille qui viennent d'être discutées ont été à l'origine de l'utilisation d'une nouvelle technique de marquage par implant visible mise en place en 1995. Cependant, l'emploi de cette technique n'a pas apporté les résultats escomptés puisque le taux de recapture a été particulièrement faible. Tout d'abord, cette faible valeur ne semble pas due à une mortalité importante de l'espèce et/ou un déplacement de population puisque 19 poissons portant des marques nouilles sont recapturés l'année suivant le marquage par implant visible. Elle ne semble pas non plus causée par une perte de l'implant dans les mois suivant le marquage. En effet, une étude actuellement en cours et réalisée sur 12 chevaines stabulés en étang montre que l'implant est toujours en place quatre mois après le marquage. En revanche, le faible taux de reprise pourrait s'expliquer en partie par une forte pression de pêche à la ligne qui s'est traduite par un nombre élevé de captures d'individus effectuées dans les semaines suivant le marquage sur le secteur étudié. Compte tenu de la difficulté à lire le marquage, les poissons marqués (absence de double marquage) n'ont pas été déclarés alors qu'habituellement les poissons marqués par marque nouille l'ont toujours été. Cette difficulté à identifier le marquage reste un obstacle pour l'utilisation d'implants visibles dans ce type d'étude, pratiquée sur une large échelle spatiale en milieu naturel. A cela, il faut ajouter la difficulté avec cette technique de marquer rapidement un grand nombre de poissons et des individus de petite taille, même pour un opérateur entraîné.

Ainsi, cette étude du suivi des populations de chevaines de la Boutonne par marquage individuel reste relativement originale tant par l'espèce concernée que par le cadre dans lequel elle s'est déroulée (conditions naturelles, rivière de taille moyenne, grande échelle spatiale à l'image d'un tronçon). Elle apporte un certain nombre d'éléments nouveaux dans la connaissance de l'écologie de l'espèce et concernant notamment la population de géniteurs, mais permet également de mettre en évidence certaines limites d'utilisation des techniques de marquage individuel mises en oeuvre depuis 1991 et qui se poursuivent actuellement sur cette rivière.

\section{REMERCIEMENTS}

Ce travail a été réalisé dans le cadre d'une étude menée en collaboration avec la Délégation Régionale du Conseil Supérieur de la Pêche de Poitiers, la Fédération Départementale des AAPPMA de Charente-Maritime et l'AAPPMA du Vairon boutonnais.

\section{BIBLIOGRAPHIE}

ALLARDI J., KEITH P., 1991. Atlas préliminaire des poissons d'eau douce de France. Secrétariat Faune Flore, MNHN, 232 p.

ARRIGNON J., 1972. Zonation piscicole de quelques cours d'eau normands. Verh. Internat. Verein. Limnol., 18, 1135-1146. 
BAGLINIĖRE J.L., LE LOUARN H., 1987. Caractéristiques scalimétriques des principales espèces de poissons d'eau douce en France. Bull. Fr. Pêche Piscic., 306, 1-39.

BAGLINIĖRE J.L., MAISSE G., LEBAIL P.Y., PREVOST E., 1987. Dynamique de population de truite commune (Salmo trutta L.) d'un ruisseau breton (France) : les géniteurs migrants. Acta Oecologia Oecol. Applic., 8, 201-215.

BAGLINIĖRE J.L., MAISSE G., LEBAIL P.Y., NIHOUARN A., 1989. Population dynamics of brown trout (Salmo trutta L.) in a tributary in Brittany (France) : spawning and juveniles. J. Fish. Biol., 34, 97-110.

BAGLINIĖRE J.L., MAISSE G., NIHOUARN A., 1993. Comparison of two methods of estimating Atlantic salmon (Salmo salar) wild production. In Production of juvenile Atlantic salmon, Salmo salar, in natural waters, GIBSON R.J. and CUTTING R.E. (Eds). Can. Spec. Publ. Fish. Aquat. Sci., 118, 189-201.

BAGLINIĖRE J.L., PORCHER J.P., 1994. Caractéristiques des stocks de reproducteurs et comportement lors de la migration génésique. In Le saumon atlantique ; biologie et gestion de la ressource, GUEGEN J.C. et PROUZET P. (Eds), IFREMER, Brest, 101-122.

BEAMISH R.J., MCFARLANE G.A., 1983. The forgotten requirement for age validation in fisheries biology. Trans. Am. Fish. Soc., 112, 735-743.

BERG O.K., BERG M., 1987. Effects of carlin tagging on the mortality and growth of sea-trout, Salmo trutta L. Fauna Norw. Ser. A, 8, 15-20.

BIANCO P.G., RECCHIA F., 1983. The Leuciscinae of the Squalius cephalus complex in Italy (Pisces, Cyprinidae). Boll. Zool., 50, 15-19.

BLANC M., BANARESCU P., GAUDET J.L., HUREAU J.C., 1971. European inland water fish. A multilingual catalogue. FAO, Fishing News Books Ltd, Londres, 23 p.

CRAGG-HINE D., JONES J.W., 1969. The growth of dace Leuciscus leuciscus (L.), roach Rutilus rutilus (L.) and chub Leuciscus cephalus (L.) in Willow Brook, Northhamptonshire. J. Fish Biol., 1, 59-82.

DELL M.B., 1968. A new fish tag and rapid, cartridge fed applicator. Trans. Am. Fish. Soc., $97,57-59$

ECONOMOU A.N., DAOULAS C., PSARRAST., 1991. Growth and morphological development of chub Leuciscus cephalus (L.) during the first year of life. J. Fish Biol., 39, 393-408.

HABASHY A.P., 1974. Age, growth, spawning and some biochemical data on the chub (Leuciscus cephalus L.) in the Roucho-Vanka creek. Zool. Listy, 23, 71-83.

HABEKOVIC D., ANICI I., SAFNER R., 1993. Dinamika rasta klena u rijeci savi. Ribart., 48, 79-88.

HANEL L., 1982. Note on the length growth of the chub (Leuciscus cephalus) in the reservoir Klicava and the river Beroumka. Vest. Cs. Spolec. Zool., 46, 241-256.

HAW F., BERGMAN R.K., FRALICK R.D., BUCKLEY R.M., BLANKENSHIP H.L., 1990. Visible implanted fish tag. Am. Fish. Soc. Symposium, 7, 311-315.

HELLAWELL J.M., 1971. The autoecology of the chub, Squalius cephalus (L.), of the river Lugg and the Afon Llynfi. Freshwat. Biol., 1, 135-148.

HICKLEY P., BAILEY R.G., 1982. Observations on the growth and production of chub Leuciscus cephalus and dace Leuciscus leuciscus in a small lowland river in southeast England. Freshwat. Biol., 12, 167-178. 
HUET M., 1949. Aperçu des relations entre la pente et les populations piscicoles des eaux courantes. Schweiz. Z. Hydrol., 11, 333-351.

KIRKA A., 1965. Age and growth of the chub Leuciscus cephalus (L.) from the Orava reservoir and its drainage area. Zool. listy, 14, 235-250.

LE LOUARN H., BAGLINIÈRE J.L., 1996. Caractéristiques biologiques et écologiques du chevaine (Leuciscus cephalus) dans quelques rivières de la façade atlantique française. Cybium, 20, 55-58.

LE LOUARN H., BAGLINIĖRE J.L., MARCHAND F., 1997. Caractéristiques biologiques et écologiques du chevaine (Leuciscus cephalus) dans quelques rivières de la façade atlantique française. Bull. Sci. Techn. INRA, Dép. Hydrobiol., 29, 27 p.

LIBOSVARSKY J., 1966. On the stability and population of chub, Leuciscus cephalus L., in a stream section. Zool. Listy, 15, 161-174.

MAISSE G., BAGLINIĖRE J.L., LEBAIL P.Y., 1987. Dynamique de population de truite commune (Salmo trutta L.) d'un ruisseau breton (France) : les géniteurs sédentaires. Hydrobiologia, 148, 123-130.

MANN R.K.H., 1976. Observations on the age, growth, reproduction and food of the chub Squalius cephalus (L.) in the river Stour, Dorset. J. Fish Biol., 8, 265-288.

MCALLISTER K.W., MCALLISTER P.E., SIMON R.C., WERNER J.K., 1992. Performance of nine external tags on hatchery-reared rainbow trout. Trans. Amer. Fish. Soc., 121, 192-198.

NICOLAS Y., PONT D., LAMBRECHTS A., 1994. Using $\gamma$-emitting artificial radionucleides, released by nuclear plants, as markers of restricted movements by chub, Leúciscus leuciscus, in a large river, the Lower Rhône. Env. Biol. Fish, 39, 399-409.

OMBREDANE D., BAGLINIĖRE J.L., 1992. Les écailles et leur utilisation en écologie halieutique. In Tissus durs et âge individuel des vertébrés, BAGLINIÈRE J.L., CASTANET J., CONAND F., MEUNIER F.J. (Ėds), ORSTOM-INRA, Paris, 151-192.

PARKER N.C., GIORGI A.E., HEIDENGER R.C., JESTER D.B., PRINCE E.D., 1990. Fishmarking techniques. Am. Fish. Soc. Symposium, 7, 879 p.

PHILIPPART J.C., 1972. Age et croissance du chevaine Leuciscus cephalus (L.) dans l'Ourthe et la Berwine. Ann. Soc. Royale Zool. Belg., 102, 47-81.

PROKES M., 1981. Seasonal growth of chub Leuciscus cephalus in the Rokytna river. Fol. Zool., 30, 371-384.

RICHARD M., 1973. Croissance et densité du chevaine (Leuciscus cephalus L.) dans la Seine. Thèse 3ème cycle, Université Paris VII, $62 \mathrm{p}$.

SPILLMANN C., 1961. Faune de France. Poissons d'eau douce. LE CHEVALIER (Ed.), Paris, $303 \mathrm{p}$.

TIMMERMANS J.A., 1957. Estimations des populations piscicoles. Trav. Stat. Rech. Eaux et Forêts, Groenendaal, Sér. $D, 21,84 \mathrm{p}$.

VITALI R., BRAGHIERI L., 1984. Population dynamics of Barbus plebejus and Leuciscus cephalus cabeda in the middle river Po (Italy). Hydrobiologia, 109, 105-124. 